\title{
Cultivar e adubação NPK na produção de tomate salada.
}

\author{
Paulo Renato Z. Santos ${ }^{1}$; Arione S. Pereira²; Cláudio José S. Freire ${ }^{2}$ \\ ${ }^{1}$ UFPEL - Colegiado de pós-graduação em agronomia, C. Postal 354, 96.010-900 Pelotas-RS; ${ }^{2}$ Embrapa Clima Temperado, C. Postal \\ 403, 96.001-970 Pelotas-RS. E-mail: arione@ cpact.embrapa.br
}

\section{RESUMO}

O objetivo deste trabalho foi estudar o efeito de cultivar, da adubação NPK e da interação desses fatores na produção de tomate salada tutorado. $\mathrm{O}$ experimento foi conduzido em Pelotas (RS) no ano agrícola 1995/96. Foram utilizadas cinco cultivares (Flora-Dade, Max, Empire, Pacific e Diva) e três níveis (2,0, 3,5 e 5,0 t/ha) de adubação NPK (3,6-7,2-10). Os tratamentos foram dispostos sob esquema fatorial em delineamento de blocos casualizados, com três repetições. As cultivares Empire e Pacific apresentaram frutos com maior peso médio do que Flora-Dade, Max e Diva. O número de frutos por planta aumentou com a elevação do nível de adubação de 2,0 para 3,5 t/ha, enquanto que o peso médio de frutos diminuiu, sem alterar a produção. As cultivares apresentaram resposta diferencial aos níveis de adubação, em relação ao peso médio de frutos.

Palavras-chave: Lycopersicon esculentum Mill., adubo, genótipo.

\begin{abstract}
Cultivar and NPK fertilization on yield of fresh market tomato.

The objective of this work was to study the effect of cultivar, NPK fertilization and the interaction on yield components of fresh market tomatoes. The experiment was carried out in Pelotas, Brazil, in 1995/96 season. Five cultivars (Flora-Dade, Max, Empire, Pacific and Diva) and three fertilization levels (2.0,3.5 and 5.0 t/ha) of NPK (3.6-7.2-10) were used. The treatments were displayed under a factorial scheme, in a randomized complete block design, with three repetitions. Empire and Pacific cultivars showed higher average fruit weight than Flora-Dade, Max and Diva. The number of fruits per plant increased with the elevation of fertilization levels from 2.0 to $3.5 \mathrm{t} / \mathrm{ha}$, while the average fruit weight diminished, without changing yield. The cultivars showed differential response to fertilization levels, in relation to average fruit weight.
\end{abstract}

Keywords: Lycopersicon esculentum Mill., fertilizer, genotype.

\section{(Aceito para publicação em 17 de janeiro de 2.001)}

$\mathrm{O}$ tomate tipo salada ocupa lugar de destaque no mercado gaúcho, ao contrário do restante do País. Em 1995, na região de Pelotas, a produção de tomate ocupou uma área de cerca de 110 ha (Universidade Católica de Pelotas, 1998). Os produtores desta região preferem as cultivares híbridas Empire e Pacific, devido ao excelente tamanho de fruto. A adubação de plantio varia de 1,5 a 2,0 t/ha das mais variadas formulações NPK (Simch, 1995), e se baseia em recomendações oriundas de outras regiões, visto que nesta não existem resultados experimentais sobre adubação em tomate tutorado.
Na região de Pelotas a recomendação de adubação para tomateiro rasteiro consiste de 100 a $120 \mathrm{~kg} / \mathrm{ha}$ de $\mathrm{N}$, $131 \mathrm{~kg} / \mathrm{ha}$ de $\mathrm{P}$ e $242 \mathrm{~kg} / \mathrm{ha}$ de $\mathrm{K}$, para solos com baixo teor de matéria orgânica e com teores limitantes de $\mathrm{P}$ e K (Comissão de Fertilidade do Solo - RS/SC, 1995). Entretanto Veduim \& Bartz (1998) testando diferentes níveis de adubação em tomate tutorado, em Santa Maria (RS), concluíram que a dose de máxima eficiência econômica situa-se em 2,87 vezes a recomendação da Rede Oficial de Laboratórios de Análise de Solo do RS e SC, ROLAS (Comissão de fertilidade do solo - RS/SC, 1995).
Para o Estado de São Paulo, Trani et al. (1996) recomendam para tomate estaqueado (12.500 pl/ha) a calagem para elevar a saturação de bases a $80 \%$ e o teor de $\mathrm{Mg}$ ao mínimo de $9 \mathrm{mmol} /$ $\mathrm{dm}^{3} ; 20$ a $30 \mathrm{t} / \mathrm{ha}$ de esterco de curral bem curtido ou 5 a $8 \mathrm{t} /$ ha de esterco de galinha; $60 \mathrm{~kg} / \mathrm{ha}$ de N, $351 \mathrm{~kg} / \mathrm{ha}$ de $\mathrm{P}$ e $250 \mathrm{~kg} / \mathrm{ha}$ de $\mathrm{K}$ no plantio, para solos de "baixa fertilidade" e $63 \mathrm{~kg} / \mathrm{ha}$ de $\mathrm{N} \mathrm{e}$ $38 \mathrm{~kg} /$ ha de $\mathrm{K}$ em cobertura, aos 15, 30, 45 e 60 dias após o transplante.

Em São Paulo, Horino et al. (1984) concluíram que as melhores doses de $\mathrm{N}$, $\mathrm{P}, \mathrm{K}$, para a produção de frutos da cv. Santa Cruz Yokota foram 300, 262 e 375 $\mathrm{kg} / \mathrm{ha}$, respectivamente. 
Nos Estados Unidos, Smith et al. (1990) encontraram resposta em peso médio de frutos das cultivares Count II, Freedom e U.S.68, inversamente proporcional às doses de NPK, enquanto a produção total de frutos com a adubação NPK, foi significativamente superior à da testemunha. A melhor adubação foi $112 \mathrm{~kg} /$ ha de N, de Pe de K.

Na Índia, Gupta \& Shukla (1977), em testes de doses de N, P e K, verificaram que o tomateiro, cv. Sioux, respondeu positivamente em produção de frutos apenas para $\mathrm{N}$ e $\mathrm{P}$. A dose que permitiu a obtenção da produtividade máxima foi de 91,5 e 59,7 kg/hade $\mathrm{N}$ e P, em 1973, e 121 e 69 kg/ha de N e P em 1974.

Dois tipos de interação, envolvendo adubação têm importância na agricultura moderna, sendo que uma delas é a interação adubação x cultivar (Raij, 1991). Filgueira et al. (1995), estudando a interação entre cultivares de batatas em diferentes locais, encontraram respostas distintas das cultivares aos locais de cultivo, o que caracteriza a existência de interação.

O objetivo deste trabalho foi obter informações referentes a cultivares e adubação NPK, para a cultura do tomateiro salada na região de Pelotas.

\section{MATERIAL E MÉTODOS}

O experimento foi conduzido no município de Pelotas, no ano agrícola de 1995/96. O solo utilizado foi classificado como Podzólico Vermelho Amarelo Distrófico, A moderado, textura argilosa (Hapludult) (Brasil, 1973). A análise do solo apresentou os seguintes resultados: argila $=18 \%$; $\mathrm{pH}=5,4$; índice $\mathrm{SMP}=59 ; \mathrm{M} . \mathrm{O} .=31 \mathrm{~g} / \mathrm{l} ; \mathrm{P}=12,5$ $\mathrm{mg} / \mathrm{l} ; \mathrm{K}=129 \mathrm{mg} / \mathrm{l} ; \mathrm{Na}=18 \mathrm{mg} / \mathrm{l} ; \mathrm{Al}=$ $0,2 \mathrm{cmol} / 1 ; \mathrm{Ca}+\mathrm{Mg}=8,5 \mathrm{cmol} / 1$. A área em que foi realizado o experimento, estava em pousio há mais de dez anos.

Foram estudados três níveis de adubação NPK de plantio e cinco cultivares de tomateiro. Os níveis de adubação foram: $72 \mathrm{~kg} /$ hade $\mathrm{N}, 328 \mathrm{~kg} /$ hade $\mathrm{P}_{2} \mathrm{O}_{5}$ e $240 \mathrm{~kg} / \mathrm{ha} \mathrm{de} \mathrm{K}_{2} \mathrm{O}$ (nível 1), $126 \mathrm{~kg} / \mathrm{ha}$ de N, $574 \mathrm{~kg} / \mathrm{ha}$ de $\mathrm{P}_{2} \mathrm{O}_{5}$ e $420 \mathrm{~kg} / \mathrm{hade}$ $\mathrm{K}_{2} \mathrm{O}$ (nível 2), $180 \mathrm{~kg} / \mathrm{ha}$ de $\mathrm{N}, 820 \mathrm{~kg} /$ ha de $\mathrm{P}_{2} \mathrm{O}_{5}$ e $600 \mathrm{~kg} / \mathrm{ha}$ de $\mathrm{K}_{2} \mathrm{O}$ (nível 3 ), obtidos pela aplicação de 2,0, 3,5 e
5,0 t/ha da formulação 3,6-7,2-10, respectivamente. $\mathrm{O}$ adubo foi aplicado em todas as parcelas, utilizando-se $2 / 3$ do total quatro dias antes do transplante e o restante, vinte dias após, por ocasião da amontoa. Foram usadas quatro cultivares híbridas (Empire, Max, Pacific e Diva) e uma de polinização aberta (FloraDade). 'Flora-Dade' e 'Pacific' apresentam hábito de crescimento determinado, 'Empire', semi-determinado, e 'Max' e 'Diva' (longa vida), indeterminado. $\mathrm{O}$ experimento foi delineado em blocos casualizados, em um esquema fatorial $3 \mathrm{x}$ 5 , com três repetições.

A semeadura foi realizada em 15 de setembro de 1995, em bandejas de isopor, e o transplante foi efetuado em 18 de outubro de 1995, quando as plantas atingiram altura igual ou superior a $10 \mathrm{~cm}$.

O preparo do solo teve início com uma aração, três meses antes do transplante, seguida de duas gradagens. Para a correção do solo, utilizou-se calcário da classe B. Este foi incorporado ao solo por ocasião da primeira gradagem, na dose de 4,0 t/ha. Quatro dias antes do transplante, foram abertos os sulcos $\mathrm{e}$ incorporados os adubos de plantio (2/3 do total). As plantas de bordadura receberam adubação equivalente ao nível mais baixo. Cada parcela foi constituída de 40 plantas, além da bordadura, sendo que apenas as 12 plantas centrais formaram a unidade de observação. As plantas foram colocadas em fileiras duplas, em espaçamentos de $0,35 \times 0,70 \times 1,10 \mathrm{~m}$, sendo $0,35 \mathrm{~m}$ entre plantas, $0,70 \mathrm{~m}$ entre fileiras simples e $1,10 \mathrm{~m}$ entre fileiras duplas.

Os demais tratos culturais (irrigação, desbrota, tutoramento, controle de invasoras, controle de pragas e de doenças) foram realizados quando se fizeram necessários, sendo uniformes em todas as parcelas e de acordo com as práticas usadas em lavouras comerciais da região.

A adubação de cobertura foi de 357 $\mathrm{kg} /$ ha de $\mathrm{N}$ e $297 \mathrm{~kg} /$ ha de $\mathrm{K}_{2} \mathrm{O}$ (mistura de sulfato de amônia e cloreto de potássio), parcelada em três aplicações aos 25, 50 e 75 dias após a amontoa, e mais uma aplicação de $793 \mathrm{~kg} / \mathrm{ha}$ de nitrato de cálcio, 100 dias após a amontoa.

A colheita foi iniciada em 26 de dezembro, sendo realizada duas vezes por semana, durante 86 dias. Os frutos de cada parcela foram colhidos, classifica- dos, contados e pesados. Frutos com diâmetro superior a $50 \mathrm{~mm}$ foram considerados como comerciais.

Os tratamentos foram avaliados pelas variáveis produção e número de frutos total e comercial. Calculou-se, ainda, a partir desses dados, o peso médio de frutos, total e comercial.

A análise estatística compreendeu a análise de variância, pelo teste F, e a comparação de médias, pelo teste de Duncan, ao nível de 5\%. Efetuou-se, também, a análise de adaptabilidade das cultivares aos níveis de adubação, através do método de Eberhart \& Russel (1966).

\section{RESULTADOS E DISCUSSÃO}

A análise da variância revelou efeito significativo dos fatores cultivar e adubo, para número total e comercial de frutos e peso médio total e comercial de frutos. A interação cultivar $\mathrm{x}$ adubo foi significativa somente para o peso médio de frutos (total e comercial). $\mathrm{O}$ fator cultivar mostrou efeito significativo ainda para produção total e comercial.

O número total e comercial de frutos por planta não diferiu estatisticamente entre os níveis de 3,5 e 5,0 t/ha de adubo, entretanto foi superior ao nível de 2,0 t/hade adubo (Tabela 1). Isto pode ser explicado pelo fato das parcelas que receberam níveis mais altos de adubo terem apresentado um maior crescimento vegetativo, possibilitando a formação de maior número de inflorescências por planta e, consequentemente um maior número de frutos.

As cultivares tiveram comportamentos distintos no que se refere aos números total e comercial de frutos. 'Diva' apresentou maior número de frutos total e comercial, seguida por 'Max' e 'FloraDade'. 'Empire' e 'Pacific' apresentaram menor número de frutos total e comercial, não diferindo entre si (Tabela 1).

'Max' foi estatisticamente superior a 'Pacific', 'Diva' e 'Flora-Dade' em produtividade total e comercial, porém não diferiu de 'Empire'. As cultivares Empire e Pacific, que são as mais plantadas na região de Pelotas, não diferiram entre si, quanto à produtividade comercial, com valores de 131,6 t/ha e 121,2 t/ha, respectivamente (Tabela 1). 
Tabela 1. Número de frutos comerciais por planta e produtividade comercial de cinco cultivares de tomate salada tutorado, em função de três níveis de adubação NPK (3,6-7,2-10). Pelotas, Embrapa Clima Temperado, 1996.

\begin{tabular}{|c|c|c|c|c|c|c|c|c|}
\hline \multirow{3}{*}{ Cultivar } & \multicolumn{4}{|c|}{ Número de frutos comerciais por planta } & \multicolumn{4}{|c|}{ Produtividade comercial de frutos (t/ha) } \\
\hline & \multicolumn{3}{|c|}{ Nível de adubação (kg/ha) } & \multirow{2}{*}{ Média } & \multicolumn{3}{|c|}{ Nível de adubação (kg/ha) } & \multirow{2}{*}{ Média } \\
\hline & 2,0 & 3,5 & 5,0 & & 2,0 & 3,5 & 5,0 & \\
\hline Diva & $30,0 a^{*}$ & 34,6 a & 31,1 a $A B$ & $31,9 a$ & $120,3 \mathrm{ab} A$ & $127,4 \mathrm{ab} \mathrm{A}$ & $119,5 \mathrm{ab} A$ & $122,4 \mathrm{bc}$ \\
\hline Max & 25,9 b $A$ & 28,6 b $A$ & $28,9 a b A$ & 27,8 b & 136,3 a $A$ & 143,1 a $A$ & 133,5 a $A$ & $137,6 \mathrm{a}$ \\
\hline Flora-Dade & 21,6 C B & 25,0 b $A B$ & 26,9 b $A$ & $24,5 \mathrm{c}$ & $108,5 b * A$ & $120,1 \mathrm{~b} \mathrm{~A}$ & 128,3 a $A$ & $119,0 \quad c$ \\
\hline Empire & 18,4 & 19,5 c A & 20,4 c A & 19,4 & $127,4 a b \quad A$ & $135,4 a b$ A & 132,2 a $A$ & $131,6 a b$ \\
\hline Pacific & 18,2 & $18,8 \subset A$ & 16,3 & 17,8 & $128,7 \mathrm{ab} A$ & $132,7 \mathrm{ab} A$ & 102,2 b $B$ & 121,2 bc \\
\hline Média & 22,8 & $25,3 \quad A$ & 24,7 & 24,3 & $124,2 \quad A$ & $131,7 \quad A$ & $123,1 \quad A$ & 126,4 \\
\hline
\end{tabular}

*/ Médias seguidas das mesmas letras, minúsculas na coluna e maiúsculas na linha, não diferem entre si pelo teste de Duncan, ao nível de $5 \%$ de probabilidade.

A análise de variância para produção total e comercial revelou efeito significativo somente para cultivar, mostrando que os genótipos estudados apresentaram diferentes necessidades nutricionais, em função, talvez de seu teto de produtividade.

A resposta não significativa, na produtividade, para doses de adubo pode ser explicada pelo fato de que os níveis testados no experimento são bem superiores à atual recomendação para a cultura no Rio Grande do Sul e Santa Catarina (Comissão de fertilidade do solo - RS/SC, 1995). Deve também ser considerado que o nível de fertilidade da área experimental foi considerado de satisfatório a bom, de acordo com os critérios de interpretação da análise do solo. Por fim, cabe lembrar que todas as parcelas experimentais receberam em cobertura, formulações contendo $\mathrm{N}$ e K. Portanto, a diferença esperada na produtividade seria devido ao $\mathrm{P}$, já que os outros dois nutrientes, pelos teores iniciais e ao adicionado em cobertura, não deveriam limitar a produtividade. Os dados mostram que a dose mais baixa de $\mathrm{P}$ já foi suficiente para se obter a máxima produtividade. Se tivesse sido usada uma testemunha sem fertilizante, provavelmente, a resposta seria outra.

A resposta não significativa para adubação, em relação à produção total e comercial de frutos (Tabela 1), está em concordância com resultados obtidos por diversos autores (Maschio \& Souza, 1982; Baungartner et al., 1988). Entretanto outros autores (Smith et al., 1990; Faria \& Pereira, 1993) encontraram resposta a níveis crescentes de adubação NPK.
Estes resultados sugerem que para solos com características semelhantes ao usado neste experimento podem ser usadas como adubação de base as doses de $72 \mathrm{~kg} / \mathrm{hade} \mathrm{N}, 328 \mathrm{~kg} / \mathrm{ha}$ de $\mathrm{P}_{2} \mathrm{O}_{5}$ e 240 $\mathrm{kg} / \mathrm{ha}$ de $\mathrm{K}_{2} \mathrm{O}(2,0 \mathrm{t} / \mathrm{ha}$ da formulação 3,6-16,4-12). Essa dose é comumente utilizada pelos tomaticultores da região (Simch, 1995). No entanto, é muito inferior à recomendada por Stevens (1997) para o RS, baseado em dados empíricos e, às recomendações para tomate tutorado em São Paulo (Trani, 1996) e para o Centro-Sul do Brasil (Filgueira, 1982). Comparando-se com a recomendação da ROLAS, verifica-se que essa dose é alta. A recomendação seria de 65 e 100 kg/ha de $\mathrm{P}$ e K, respectivamente. Cabe ressaltar, entretanto, que a recomendação da ROLAS é baseada na cultura de tomate rasteiro, que utiliza o menor nível tecnológico, requerendo menor quantidade de nutrientes.

As médias de produção total e comercial de frutos $(137,3 \mathrm{t} /$ ha e $126,4 \mathrm{t} /$ ha, respectivamente) obtidas neste trabalho demonstram que a cultura tem um potencial produtivo bem maior que a produção média (60 t/ha) obtida na região de Pelotas (Universidade Católica de Pelotas, 1998), sendo bastante superior, entretanto, à média nacional $(43,8$ t/ha) e do Rio Grande do Sul (31,8 t/ha) (Anuário Estatístico do Brasil, 1996).

As cultivares Max, Empire e Pacific tiveram pesos médios total e comercial de frutos diminuídos com o incremento na quantidade de adubo de 2,0 para 5,0 t/ha, concordando com os resultados obtidos por Gupta \& Shukla (1977), para NP, Maschio \& Souza (1982), para PK, e Smith et al. (1990), para NPK.
As cultivares Empire e Pacific destacaram-se com relação ao peso médio de frutos (total e comercial), sendo estatisticamente superiores às demais. 'FloraDade' e 'Max' apresentaram comportamento intermediário. Entre as cultivares testadas, Diva foi a que apresentou menor peso médio total e comercial.

O peso médio dos frutos das cultivares Empire e Pacific observado neste trabalho é um pouco inferior aos relatados por Peixoto et al. (1996), para a cultivar Empire, no Triângulo Mineiro, e por Silva Júnior et al. (1995), para a cultivar Pacific, no Litoral Catarinense.

Os resultados de número de frutos (Tabela 1) e de peso médio (Tabelas 2) mostram que houve diferenças entre as cultivares, independente do nível de adubação, sugerindo que essas são características próprias de cada cultivar. No entanto, cultivares que produzem grande número de frutos, apresentam também menor peso médio, confirmando os resultados de Maschio \& Souza (1982). Valores semelhantes foram obtidos por Postingher (1995), em Pelotas, com médias entre 27,5 e 29,2 frutos por planta, para cultivares de hábito de crescimento indeterminado.

O peso médio de frutos é uma característica importante, pois o principal critério de classificação do tomate é baseado no tamanho. Produzindo frutos de tamanho reduzido, o tomaticultor tem dificuldade em colocar o produto no mercado, de forma competitiva.

Como a interação cultivar $\mathrm{x}$ adubo foi significativa para a variável peso médio de frutos, realizou-se análise de adaptabilidade, pelo método de Eberhart 
Tabela 2. Peso médio total e de frutos comerciais de cinco cultivares de tomate salada tutorado, em função três níveis de adubação NPK (3,6-7,2-10). Pelotas, Embrapa Clima Temperado, 1996.

\begin{tabular}{|c|c|c|c|c|c|c|c|c|c|c|}
\hline \multirow{4}{*}{$\begin{array}{c}\text { Cultivar } \\
\text { Flora-Dade }\end{array}$} & \multicolumn{6}{|c|}{ Peso médio total (g/fruto) } & \multicolumn{4}{|c|}{ Peso médio de frutos comerciais ( $\mathrm{g} /$ fruto) } \\
\hline & \multicolumn{5}{|c|}{ Nível de adubação (kg/ha) } & \multirow{2}{*}{ Média } & \multicolumn{3}{|c|}{ Nível de adubação (kg/ha) } & \multirow{2}{*}{ Média } \\
\hline & \multicolumn{2}{|c|}{2,0} & 3,5 & \multicolumn{2}{|c|}{5,0} & & 2,0 & 3,5 & 5,0 & \\
\hline & $142 k$ & $b^{*} A$ & $135 \mathrm{~b} A$ & $134 k$ & b A & $137 \mathrm{~b}$ & 158 b $A$ & $150 \mathrm{~b} \quad \mathrm{~A}$ & 150 b A & $153 \mathrm{~b}$ \\
\hline Max & 152 & b $A$ & b $A B$ & $134 \mathrm{k}$ & b $B$ & $143 \mathrm{~b}$ & 166 b A & 158 b $A B$ & 146 b $B$ & $157 \mathrm{~b}$ \\
\hline Empire & $200 a$ & $A$ & 196 a A & $180 \mathrm{a}$ & B & 192 a & 218 a $A$ & 217 a $A$ & 204 a B & $213 a$ \\
\hline Pacific & $207 a$ & A & 202 a A & $171 \mathrm{a}$ & B & $193 a$ & $222 a^{\star} A$ & 222 a & 197 a $B$ & $214 a$ \\
\hline Diva & 116 & C A & 106 & 111 & $c A$ & $111 \mathrm{C}$ & c A & $C A$ & 122 & $121 \mathrm{C}$ \\
\hline Média & 163 & A & 157 & 146 & C & 155 & 178 & 172 & 164 & 171 \\
\hline
\end{tabular}

*/ Médias seguidas das mesmas letras, minúsculas na coluna e maiúsculas na linha, não diferem entre si pelo teste de Duncan, ao nível de $5 \%$ de probabilidade.

Tabela 3. Análise de adaptabilidade, quanto ao peso médio de frutos (g/fruto), de cinco cultivares de tomate salada tutorado, em função do uso de três níveis de adubação NPK (3,67,2-10). Pelotas, Embrapa Clima Temperado, 1996.

\begin{tabular}{lllcc}
\hline \multirow{2}{*}{ Cultivar } & \multicolumn{2}{c}{ Peso médio total } & \multicolumn{2}{c}{ Peso médio comercial } \\
\cline { 2 - 5 } & Média & $\boldsymbol{b}^{\boldsymbol{*}}$ & Média & $\boldsymbol{b}$ \\
\hline Pacific & 193,6 & 2,07 & 213,8 & 1,77 \\
Empire & 192,0 & 1,17 & 213,3 & 0,98 \\
Max & 143,4 & 1,05 & 156,4 & 1,42 \\
Flora-Dade & 136,9 & 0,47 & 152,6 & 0,54 \\
Diva & 110,7 & 0,57 & 121,0 & 0,79 \\
\hline
\end{tabular}

*/ Coeficiente de regressão linear obtido da regressão da média de peso médio de frutos das cultivares na média de peso médio de frutos dos níveis de adubação para cada cultivar $(b>$ 1 significa adaptação restrita aos níveis baixos de adubação; $b<1$ indica adaptação aos níveis altos de adubação; $b=1,0$ indica adaptação ampla aos diferentes níveis de adubação).

\& Russel (1966). Nesta análise de regressão, o coeficiente $(b)$ indica a resposta dos genótipos a condições do ambiente. Para cada cultivar, foi calculada a regressão linear do peso médio de frutos em relação à média de peso de frutos de todas as cultivares (Tabela 3 ). 'Pacific' apresentou $b>1$, que indica adaptação restrita aos níveis baixos de adubação. 'Flora-Dade' e 'Diva' $(b<$ 1) mostraram-se mais adaptadas a doses altas de adubação. Estes genótipos não foram afetados de modo significativo no peso médio com o aumento dos níveis de adubação.

A cultivar Empire, para peso médio total e comercial, e Max, para peso médio total, apresentaram valores de $b$ muito próximos à unidade, sugerindo ampla adaptabilidade às diferentes doses de adubo (Tabela 3).

Os resultados envolvendo 'Pacific', melhor adaptada a níveis mais baixos de adubação, e 'Empire', de ampla adaptabilidade, explicam o bom desempenho dessas cultivares na região de Pelotas, onde se utiliza níveis de adubação de baixos a médios, quando comparados aos níveis testados neste trabalho.

Nas condições do presente trabalho, as cultivares Empire e Pacific foram superiores à Flora-Dade, Max e Diva, quanto ao peso médio total e comercial de frutos. O número de tomates produzido por planta aumentou com o nível de adubação, enquanto que o peso médio de frutos diminuiu, porém sem alterar a produção. As cultivares apresentaram resposta diferencial aos níveis de adubação para peso médio de frutos.

\section{LITERATURA CITADA}

ANUÁRIO ESTATÍSTICO DO BRASIL. Rio de Janeiro: $I B G E$, v. 56, 1996.

BAUNGARTNER, J.G.; HAAG, H.P.; OLIVEIRA, G.D.; PERECIN, D. Tolerância de cultivares de tomateiro (Lycopersicon esculentum Mill.) ao alumínio e ao manganês. In: HAAG, H.P.; MINAMI, K. Nutrição mineral em hortaliças. Campinas: Fundação Cargill, 1988. 538 p.
BRASIL. MINISTÉRIO DA AGRICULTURA Levantamento de reconhecimento dos solos do Estado do Rio Grande do Sul. Recife: 1973. 431 p. (Boletim Técnico, 30).

COMISSÃO DE FERTILIDADE DO SOLO $\mathrm{RS} / \mathrm{SC}$. Recomendações de adubação e calagem para os Estados do Rio Grande do Sul e Santa Catarina. Passo Fundo: SBCS, 1995. $224 \mathrm{p}$.

EBERHART, S.A.; RUSSEL, W.A. Stability parameters for comparing varieties. Crop Science, v. 6, p. 36-40, 1966.

FARIA, C.M.B.; PEREIRA, J.R. Movimento do fósforo no solo e seu modo de aplicação no tomateiro rasteiro. Pesquisa Agropecuária Brasileira, Brasília, v. 28, p. 1363-1370, 1993.

FILGUEIRA, F.A.R. Manual de Olericultura: cultura e comercialização de hortaliças. São Paulo: Agronômica Ceres, v. 2, 1982. 357 p.

FILGUEIRA, R.A.R.; BANZATTO, D.A.; CHURATA-MASCA, M.G.C.; CASTELLANE, P.D. Interação genótipo $x$ ambiente em batata. Horticultura Brasileira, Brasília, v. 13, n. 2, p. 134-141, nov. 1995.

GUPTA, A.; SHUKLA, V. Response of tomato (Lycopersicon esculentum Mill.) to plant spacing, nitrogen, phosphorus and potassium fertilization. Indian Journal of Horticulture, Bangalore, v. 34, p. 270-276, 1977.

HORINO, Y.; SHIROZU, R.; SHIROSE, I. Ensaio de níveis de adubação para tomateiro em Itapetininga - SP. In: CONGRESSO BRASILEIRO DE OLERICULTURA, 24, 1984 Jaboticabal. Resumos... Jaboticabal: FCAV, 1984. p. 8.

MASCHIO, L.M.A.; SOUZA, G.F. Adubação básica, nitrogênio em cobertura. Pesquisa Agropecuária Brasileira, Brasília, v. 17, p. 1309-1315, 1982.

PEIXOTO, J.R.; FARIA, V.R.C.A.; RODRIGUES, F.A.; SILVA, R.P.; RAMOS, R.S.; JULIATTI, F.C. Avaliação de genótipos de tomate tipo "salada", no período das águas, em Araguari - MG. In: CONGRESSO BRASILEIRO DE OLERICULTURA, 36, 1996, Rio de Janeiro. Resumos... Brasília: Horticultura Brasileira, Brasília, 1996, v. 14 n. 1, p. 106.

POSTINGHER, D. Respostas agronômicas e consumo hídrico da cultura do tomateiro cultivado em estufa plástica. Pelotas: UFPEL, 1995. 59 p. (Dissertação mestrado).

RAIJ, B. VAN. Fertilidade do solo e adubação. São Paulo: Ceres/Potafos, 1991. 343 p. 
SILVA JUNIOR, A.A.; VIZZOTTO, V.J.; STUKER, H. Cultivares de tomate para o Baixo Vale do Itajaí. Agropecuária Catarinense, Florianópolis, v. 8, n. 4, p. 35-39, 1995.

SIMCH, T.L. Produção e comercialização de tomate tipo salada em Pelotas, RS. Pelotas: UFPEL, 1995. 76 p. (Dissertação mestrado).

SMITH, C.B.; DEMCHAK, K.T.; FERRETI, P.A. Fertilizer placement effects on growth and nutrient uptake on sweet corn, snapbeans, tomatoes and cabbage. Communications in soil science and plant analysis, New York, v. 21, p. $107-123,1990$.
STEVENS, J. A cultura do tomate tutorado. $\mathrm{Pa}$ lestra apresentada para produtores da Colônia Santa Helena, Pelotas. 25/Julho/1997.

TRANI, P.E.; NAGAI H.; PASSOS, F.A. Tomate (estaqueado). In: RAIJ, B. VAN; CANTARELLA, H.; QUAGGIO, J.A.; FURLANI, A.M.C. Recomendações de adubação e calagem para o Estado de São Paulo. 2 ed. Campinas: IAC, 1996. p. 184, 285 p. (Boletim Técnico, 100).
UNIVERSIDADE CATÓLICA DE PELOTAS. Instituto Técnico de Pesquisa e Assessoria. Banco de dados da Zona Sul - RS. Pelotas: EDUCAT, n. 9, 1998. 209 p.

VEDUIM, J.V.R.; BARTZ, H.R. Fertilidade do solo e rendimento do tomateiro em estufa de plástico. Ciência Rural, Santa Maria, v. 28, n. 2, p. 229-233, 1998 